\title{
Aplicación de un programa de fuerza con carga excéntrica en la readaptación de una lesión de ligamento lateral interno de la rodilla. Estudio de caso \\ Effect of an eccentric load resistance training program on the rehabilitation of medial collateral ligament injuries. A case study \\ *Javier Raya-González, **Pedro Gómez Piqueras, ***Javier Sánchez-Sánchez \\ *Universidad Isabel I, Burgos (España), **Universidad de Castilla La Mancha (España), ***Universidad Pontificia de Salamanca (España)
}

\begin{abstract}
Resumen. El objetivo del presente estudio fue analizar el efecto de un programa de entrenamiento de fuerza con carga excéntrica basado en el ejercicio squat ( $1 / 2$ squat y squat lateral) ejecutado en un dispositivo de inercia rotacional, sobre la potencia de la musculatura del tren inferior de un jugador de fútbol durante la readaptación de una lesión del ligamento lateral interno (LLI) de la rodilla. Métodos: Durante 4 semanas (3 sesiones semanales) el futbolista llevó a cabo el programa de entrenamiento de fuerza con carga excéntrica propuesto en ambas extremidades. Antes, durante y después del proceso de intervención se valoró la potencia del tren inferior de manera bilateral y unilateral, y se obtuvieron datos relativos a la potencia media, potencia pico, trabajo y duración de la repetición, tanto en fase concéntrica como excéntrica. Resultados: Se obtuvieron mejoras significativas (pd».05) en la potencia media y pico (bilateral y unilateral) tanto en la fase concéntrica como excéntrica. Además se redujeron las asimetrías en todas las variables analizadas relativas a la potencia. Conclusiones: La aplicación de un programa de fuerza ejecutado en un dispositivo de inercia rotacional durante el proceso de recuperación funcional produce un incremento de la potencia muscular del tren inferior y disminuye la asimetría provocada por la lesión deportiva, que se refleja en la disminución del periodo de recuperación.
\end{abstract}

Palabras clave. Rehabilitación, potencia, lesión, fútbol.

\begin{abstract}
The aim of this study was to analyse the effect of an eccentric-load training program based on squat exercises ( $1 / 2$ squat and lateral squat) on the lower-body power of a football player during the rehabilitation process of a medial collateral ligament injury (MCL). Methods: During 4 weeks (3 sessions a week) the soccer player followed the eccentric-load training program proposed on both legs. Before, during, and after the intervention, lowerbody power was evaluated bilaterally and unilaterally, and data was obtained on the mean power, peak power, work, and duration, in both concentric and eccentric phases. Results: Significant improvements ( $\mathrm{p} d » .05)$ in mean and maximal power (bilateral and unilateral) were obtained in both concentric and eccentric phases. Asymmetries related to power have also been reduced. Conclusions: the application of an eccentric-overload training program during the functional recovery process results in an increase in the lower-body power and reduces the asymmetry caused by a sports injury, which is reflected in the decrease in the recovery period.
\end{abstract}

Keywords. Rehabilitation, maximal power output, injury, soccer.

\section{Introducción}

El fútbol es un deporte de contacto que demanda en el jugador una elevada participación psicológica, técnico-táctica y física (Carling, Bloomfield, Nelsen, \& Reilly, 2008; Owen, Wong, McKenna, \& Dellal, 2011). Respecto a esta última, la sucesión aleatoria de acciones de alta intensidad junto a períodos de recuperación caracteriza la intervención condicional del jugador durante la competición (Ade, Fitzpatrick, \& Bradley, 2016). Estas acciones de alta intensidad junto a las oscilaciones en la carga de entrenamiento (Gabbett, 2016) convierten a los futbolistas en los deportistas de equipo más susceptibles de sufrir lesiones durante la práctica deportiva (Van Winckel, Helsen, McMillan, Tenney, Meert \& Bradley, 2014). Además, durante los partidos se produce en las extremidades inferiores una sobrecarga de las estructuras anatómicas que incrementa aún más el riesgo de sufrir una lesión muscular o articular (Miyamoto, Bosco \& Sherman, 2009).

El ligamento lateral interno (LLI) de la rodilla es una estructura articular con un elevado riesgo de lesión en deportes de contacto (Roach et al., 2014). En equipos de fútbol europeos de alto nivel, el ratio para este tipo de lesión es aproximadamente de 0.33 les $/ 1000 \mathrm{~h}$ de exposición (lesiones de LLI/1000h) (Noya, Gómez-Carmona, Gracia-Marco, Moliner-Urdiales, \& Sillero-Quintana, 2014). Además, para un equipo de 25 jugadores se pueden esperar aproximadamente 2 lesiones del LLI de la rodilla cada temporada (Lundblad, Waldén, Magnusson, Karlsson \& Ekstrand, 2013). La mayoría de estas lesiones del LLI no requieren cirugía y son abordadas con tratamiento conservador (Macmahon, 2007). Esta intervención implica la inmovilización y posterior rehabilitación por un plazo de 3-4 semanas (Phisitkul, James, Wolf \& Amendola, 2006). La media de días de baja por lesión del futbolista con esguince de LLI de rodilla es de $23 \pm 23$ días, existiendo un $28.3 \%$ de estas lesiones que requieren un proceso de recuperación mayor a 28 días (Lundblad et al., 2013).

Fecha recepción: 10-03-17. Fecha de aceptación: 03-07-17

Javier Raya-González

rayagonzalezjavier@gmail.com
Los días de baja por lesión causan desentrenamiento y disminución del rendimiento deportivo (Ekstrand, Hägglund \& Walden, 2011). Entre las causas que provocan esta desadaptación destacan el descenso en la capacidad cardiovascular, el aumento del cociente respiratorio, la disminución del $\mathrm{VO}_{2 \max }$ y el declive del umbral láctico (Ronconi \& AlveroCruz, 2008). Además existe una pérdida en el rendimiento neuromuscular, que se materializa en una menor capacidad para aplicar fuerza durante las tareas deportivas (Andersen et al., 2005). La pérdida de fuerza provocada por el desentrenamiento suele derivar en asimetrías funcionales en las extremidades inferiores (Fousekis, Elias, \& Vagenas, 2010). Estas asimetrías provocan modificaciones en la mecánica del gesto deportivo que disminuyen el rendimiento e incrementan el riesgo de lesión (Menzel et al., 2013). Esto es debido a que la extremidad más débil tiene dificultades para tolerar la elevada presión a la que se ve sometida durante las acciones de salto, cambio de dirección y frenada propias del deporte (Hoffman, Ratamess, Klatt, Faigenbaum, \& Kang, 2007). Esta circunstancia hace que uno de los objetivos principales de los programas de readaptación físico deportiva en futbolistas deba ser la reducción de las asimetrías causadas por las lesiones (Núñez, Lancho \& Ramírez, 2016).

El entrenamiento de fuerza con carga excéntrica genera grandes tensiones en la musculatura que se han mostrado efectivas para la prevención de lesiones (Askling, Karisson, Thorstensson, 2003; De Hoyo, Pozzo, Sañudo, Carrasco, Gonzalo-Skok, Domínguez-Cobo \& Morán-Camacho, 2015). Este estímulo de entrenamiento puede ser aplicado a través de los dispositivos de inercia rotacional (Romero, Feria, Sañudo, De Hoyo \& Del Ojo, 2014; Tous-Fajardo, Maldonado, Quintana, Pozzo \& Tesch, 2006). Estos dispositivos son una estrategia de entrenamiento útil en el proceso de readaptación físico-deportiva de futbolistas por su incidencia sobre factores determinantes de la condición física específica (Romero-Rodríguez, Gual \& Tesch, 2011). Un estudio realizado con 15 futbolistas profesionales mostró mejoras significativas en los niveles de fuerza excéntrica y concéntrica (19 y 15\% respectivamente) de la extremidad inferior tras 10 semanas de un programa de entrenamiento que incluía ejercicios con sobrecarga excéntrica aplicada con el dispositivo de inercia rotacional YOYO-Hamstring (Askling et al., 2003). Resultados similares fueron observados con 
futbolistas juveniles que complementaron su entrenamiento regular con 2 sesiones/semana de fuerza con carga excéntrica, obteniendo mejoras en habilidades específicas como el salto y el sprint (de Hoyo et al., 2015).

Los objetivos del presente estudio de caso fueron: (1) conocer el efecto de un programa de entrenamiento de fuerza con carga excéntrica ejecutado con dispositivos de inercia rotacional, sobre la potencia del tren inferior, y (2) comprobar la eficacia del programa de entrenamiento de fuerza sobre la reducción de las asimetrías funcionales de fuerza de la extremidad inferior causadas por la lesión del LLI de la rodilla del futbolista lesionado.

\section{Metodología}

\section{Descripción del caso}

El futbolista participante es un varón de 22 años, $171.7 \mathrm{~cm}$ de estatura, $70.2 \mathrm{~kg}$ de peso y $7.8 \%$ de masa grasa. El jugador actúa en la demarcación de lateral derecho del equipo filial de un club de fútbol profesional ( $1^{\mathrm{a}}$ División Española) y compite en la categoría $2^{\mathrm{a}}$ División B. El modelo de programación utilizado por el equipo filial se basó en la microestructuración, siendo la semana tipo de entrenamiento (competición domingo-domingo) la siguiente: lunes (recuperación), martes (descanso), miércoles (fuerza-espacios reducidos), jueves (resistenciaespacios amplios), viernes (recuperación-estrategia) y sábado (activación pre-partido). Durante uno de los entrenamientos del equipo el futbolista sufrió un valgo forzado en su rodilla derecha debido a la colisión con otro jugador. La primera exploración física reveló hinchazón y sensibilidad ósea en el cóndilo femoral interno así como cierta laxitud en el LLI. El tratamiento prescrito para las primeras 48 horas consistió en reposo, hielo, compresión y elevación (RICE). Una vez finalizado este plazo, el futbolista fue sometido a una resonancia magnética que reveló un esguince de grado II en el LLI de su rodilla derecha. Tras la consulta con el médico del club se decidió seguir un tratamiento conservador utilizado con éxito en las lesiones el LLI de la rodilla (Machamon, 2007).

Una vez conocido el diagnóstico se programó un proceso de readaptación dividido en 5 fases (Tabla 1): tratamiento médico, aproximación, orientación, preoptimización y optimización (Lalín, 2008).

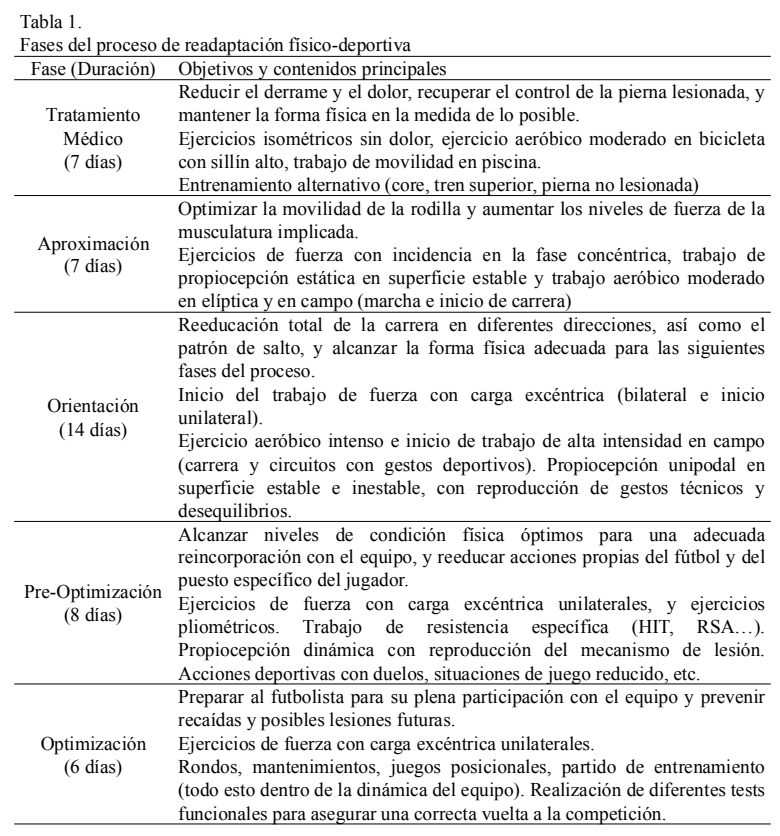

Antes de iniciar el estudio el participante firmó el correspondiente consentimiento informado donde se indicaban los derechos y obligaciones derivados de su participación. Los procedimientos fueron realizados de acuerdo a los estándares éticos recomendados por la Declaración de Helsinki (2013).

\section{Valoración}

Tras superar de manera satisfactoria las dos primeras fases del proceso de readaptación, se dio paso a la fase de orientación. La intervención duró cuatro semanas (entre los meses de febrero y marzo, correspondientes al periodo competitivo). En la primera semana el futbolista llevó a cabo el pre-test bilateral, y durante la segunda semana tuvo lugar la realización del pre-test unilateral. Una vez que finalizó el programa de entrenamiento propuesto se llevó a cabo el post-test, de manera bilateral y unilateral siguiendo el mismo procedimiento que en el pre-test.

Para medir la potencia del tren inferior se utilizó un dispositivo de inercia rotacional (K-Box 3, Exxentric ${ }^{\circledR}$, Stockholm, Suecia) por medio de un encoder rotatorio (SmartCoach ${ }^{\mathrm{TM}}$ Power Encoder, SmartCoach Europe AB, Stockholm, Suecia). Tras un calentamiento estandarizado (parte general, basada en tres minutos de ejercicios aeróbicos y tres minutos de ejercicios de movilidad articular; parte específica, en la que se incluyeron cinco repeticiones del ejercicio de media sentadilla ejecutado de manera bilateral a velocidad no máxima, tres repeticiones del ejercicio de media sentadilla ejecutado de manera unilateral a velocidad no máxima y dos series de ocho repeticiones a velocidad progresiva del ejercicio que se iba a realizar en la sesión posterior), el futbolista ejecutó dos series de seis repeticiones del ejercicio media sentadilla de manera bilateral (inercia $0.050 \mathrm{~kg} / \mathrm{m}^{2}$ ) y dos series de seis repeticiones del ejercicio squat lateral con cada pierna (inercia $0.025 \mathrm{~kg} / \mathrm{m}^{2}$ ), con un descanso de cuatro minutos entre cada intento.

Se midió la potencia media y potencia pico (bilateral y unilateral) en ambas fases del movimiento (concéntrica y excéntrica), el trabajo realizado en cada repetición y la duración de la misma. Estos valores fueron usados para el posterior análisis estadístico, seleccionando el mejor resultado obtenido en cada test.

Además, se calculó el porcentaje de asimetría entre ambas piernas como el cociente de la diferencia entre la pierna no lesionada (NL) y la pierna lesionada $(\mathrm{L})$ entre el de la pierna no lesionada $(\mathrm{NL})$ expresado en porcentaje: [(NL-L)/NL]x100 (Gustavsson et al., 2006). Los valores positivos indicaron que la variable tiene un valor superior en NL (Núñez et al., 2016).

La duración de la lesión se consideró como el tiempo que transcurrió entre el día que se produjo la lesión y el día en que el futbolista participó de manera plena en la sesión de entrenamiento y estaba en disposición de ser convocado (Hägglund, Waldén, Bahr, \& Ekstrand, 2005).

\section{Intervención}

El programa de entrenamiento de fuerza con carga excéntrica tuvo una duración de cuatro semanas, realizando tres sesiones/semana los lunes, miércoles y viernes. De las doce sesiones de fuerza, cinco tuvieron un carácter bilateral $\left(1 / 2\right.$ sentadilla con inercia $\left..050 \mathrm{~kg} / \mathrm{m}^{2}\right)$ y siete tuvieron orientación unilateral (squat lateral con inercia $.025 \mathrm{~kg} / \mathrm{m}^{2}$ ). Todas se realizaron empleando el dispositivo de inercia rotacional $K$ Box 3 (Exxentric ${ }^{2}$, Stockholm, Suecia). Cada sesión de entrenamiento consistió en cuatro series de ocho repeticiones $(4 \mathrm{x} 8$; las dos primeras se utilizaron para la aceleración del volante de inercia) con tres minutos de recuperación. Durante la ejecución se pidió al jugador que realizase el movimiento a la máxima velocidad. Previamente a las sesiones de fuerza, el futbolista realizó el mismo calentamiento descrito durante la realización de los test de valoración.

\section{Análisis estadístico}

Se realizó un análisis descriptivo de las variables, siendo los datos presentados en forma de medias y desviaciones estándar (SD). Los datos relativos al índice de simetría se presentaron en porcentaje (\%). Se aplicaron pruebas de bondad de ajuste de Shapiro-Wilk para determinar la normalidad de cada variable de estudio.

Para determinar el efecto del programa de fuerza con sobrecarga excéntrica se aplicaron pruebas de contraste para muestras relacionadas con los valores pre test y post test (Núñez et al., 2016). En aquellas variables normales se aplicaron estadísticos paramétricos (Prueba T), 
mientras que en aquellas variables que no presentaron normalidad se utilizaron estadísticos no paramétricos (U de Mann Whitney). Adicionalmente, se calculó el tamaño del efecto (TE) a través de la prueba d de Cohen (Cohen, 1988) utilizando la siguiente fórmula, al tratarse de un análisis intra-grupo (ES= (Media Post test- Media Pre test)/ SD Pre test). La interpretación de d se realizó utilizando la escala para «altamente entrenados» (Owen et al., 2013), cuyos valores fueron: $<.25$ (trivial), $.25-.50$ (pequeño), .50-1.0 (moderado) y $>1.0$ (grande). El cambio en asimetrías se ha analizó en valores absolutos y se consideró como situación de alto riesgo aquellos valores de asimetría superiores al 15\% (Petisco, Carretero y Sánchez-Sánchez, 2016). El análisis estadístico de los datos se llevó a cabo usando el software SPSS para Windows Versión 21.0(SPSS Inc., Chicago, Illinois, USA), con p d».05 para diferencias estadísticamente significativas.

\section{Resultados}

\section{Evaluación bilateral}

En la Tabla 2 se presentan los valores de potencia, trabajo y duración de la repetición relativos al test bilateral. Se obtuvieron mejoras significativas $(p<.05)$ en todas las variables de potencia analizadas, tanto en la fase concéntrica del movimiento (TE grande) como en la fase excéntrica (TE grande), analizadas de manera aislada. Estas mejoras también se vieron reflejadas cuando se analizaron ambas fases de manera conjunta (TE grande). Además, el trabajo total aumentó de manera significativa (pd».05) en el post-test respecto al pre-test (TE grande) y la duración total de la repetición se redujo de manera significativa ( $\mathrm{pd} \gg .05$; TEgrande).

Tabla 2.

(media + SD) después del programa de entrenamiento de fuerza

\begin{tabular}{|c|c|c|c|c|c|}
\hline Bilateral & Pre-test & Post-test & $\%$ cambio & \multirow{2}{*}{$P$} & \multirow{2}{*}{$\begin{array}{cc}\text { ES } \\
3.65\end{array}$} \\
\hline Potencia Media CON (W) & $221.72 \pm 49.58$ & $402.88 \pm 18.47^{*}$ & 81.71 & & \\
\hline Potencia Media ECC (W) & $220.67 \pm 60.59$ & $364.67 \pm 49.63^{*}$ & 65.26 & .001 & 2.38 \\
\hline Potencia Media Total (W) & $442.38 \pm 107.18$ & $767.55 \pm 40.81^{*}$ & 73.50 & .0001 & 3.03 \\
\hline Potencia Pico CON (W) & $477.17 \pm 156.2$ & $844.75 \pm 36.50^{*}$ & 77.03 & .0001 & 2.35 \\
\hline Potencia Pico ECC (W) & $545.18 \pm 142.98$ & $886.85 \pm 116.87^{*}$ & 62.67 & .001 & 2.39 \\
\hline Potencia Pico Total (W) & $1022.35 \pm 293.1$ & 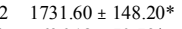 & 69.37 & .0001 & 2.42 \\
\hline Trabajo Total (J) & $535.73 \pm 105.3$ & $696.18 \pm 70.73^{*}$ & 29.95 & .01 & 1.52 \\
\hline Duración Total (s) & $2.45 \pm .13$ & $1.83 \pm .12 *$ & -25.54 & .001 & 4.8 \\
\hline \multicolumn{6}{|c|}{$\begin{array}{l}\text { Nota: CON: concéntrica; ECC: excéntrica. Niveles de significación * p } \leq .05 \text {. ES: tamaño del } \\
\text { efecto }\end{array}$} \\
\hline \multicolumn{6}{|c|}{$\begin{array}{l}\text { Tabla } 3 . \\
\text { Cambios en el rendimiento unilateral de la pierna no lesionada (media } \pm \text { SD) después del } \\
\text { programa de entrenamiento de fuerza. }\end{array}$} \\
\hline Unilateral & Pre-test & Post-test & 6 cambio & $\mathrm{P}$ & ES \\
\hline Potencia Media CON (W) & $197.93 \pm 19.55$ & $312.20 \pm 44.03^{*}$ & 57.73 & .0001 & 5.84 \\
\hline Potencia Media ECC (W) & $169.42 \pm 12.30$ & $239.95 \pm 42.05 *$ & 41.63 & .003 & 5.73 \\
\hline Potencia Media Total (W) & $367.35 \pm 20.09$ & $552.15 \pm 85.18^{*}$ & 50.31 & .0001 & 5.20 \\
\hline Potencia Pico CON (W) & $444.90 \pm 36.47$ & $566.18 \pm 73.74 *$ & 27.26 & .005 & 3.33 \\
\hline Potencia Pico ECC (W) & $479.60 \pm 118.86$ & $460.65 \pm 138.71$ & -3.95 & .805 & 0.16 \\
\hline Potencia Pico Total (W) & $924.50 \pm 127.42$ & $1026.83 \pm 204.15$ & 11.07 & .322 & 0.80 \\
\hline Trabajo Total (J) & $322.15 \pm 14.34$ & $329.27 \pm 13.42$ & 2.21 & .396 & 0.50 \\
\hline Duración Total (s) & $1.77 \pm .08$ & $.95 \pm 0.05^{*}$ & -46.24 & .0001 & 5.43 \\
\hline \multicolumn{6}{|c|}{$\begin{array}{l}\text { Nota: CON: concéntrica; ECC: excéntrica. Niveles de significación * } \mathrm{p} \leq .05 \text {. ES: tamaño del } \\
\text { efecto }\end{array}$} \\
\hline \multirow{2}{*}{\multicolumn{6}{|c|}{$\begin{array}{l}\text { Tabla } 4 \text {. } \\
\text { Cambios en el rendimiento unilateral de la pierna lesionada (media } \pm \text { SD) después del programa } \\
\text { de entrenamiento de fuerza. }\end{array}$}} \\
\hline & & & & & \\
\hline Unilateral & Pre-test & Post-test & 6 cambio & $\mathrm{P}$ & ES \\
\hline Potencia Media CON (W) & $142.42 \pm 27.88$ & $321.18 \pm 20.55^{*}$ & 125.52 & .0001 & 6.41 \\
\hline Potencia Media ECC (W) & $122.52 \pm 38.33$ & $238.45 \pm 62.14^{*}$ & 94.63 & .003 & 3.02 \\
\hline Potencia Media Total (W) & $264.93 \pm 63.46$ & $559.63 \pm 69.13 *$ & 111.24 & .0001 & 4.64 \\
\hline Potencia Pico CON (W) & $267.70 \pm 70.82$ & $537.82 \pm 109.05^{*}$ & 100.90 & .0001 & 3.81 \\
\hline Potencia Pico ECC (W) & $264.87 \pm 47.33$ & $492.97 \pm 62.26^{*}$ & 86.12 & .0001 & 4.82 \\
\hline Potencia Pico Total (W) & $532.57 \pm 115.17$ & $1030.78 \pm 144.14 *$ & 93.55 & .0001 & 4.33 \\
\hline Trabajo Total (J) & $250.98 \pm 61.25$ & $331.42 \pm 47.24 *$ & 32.05 & .03 & 1.31 \\
\hline Duración Total (s) & $1.93 \pm 10$ & $.92 \pm 0.06^{*}$ & -52.25 & .0001 & 5.48 \\
\hline \multicolumn{6}{|c|}{$\begin{array}{l}\text { Nota: CON: concéntrica; ECC: excéntrica. Niveles de significación * } \mathrm{p} \leq .05 \text {. ES: tamaño del } \\
\text { efecto }\end{array}$} \\
\hline$\%$ de asimetría & Pre-test & $\begin{array}{l}\text { - Post-test } \\
39.83\end{array}$ & 44.77 & 42.39 & \\
\hline \multicolumn{6}{|l|}{40} \\
\hline $30 \quad 28.05$ & 27.8 & & & & \\
\hline 20 & & & & & \\
\hline 10 & & & & & \\
\hline 0 & 63 & 5.01 & & & \\
\hline-10 & & -1.36 & & & -.38 \\
\hline $\begin{array}{l}\text { Potencia } \\
\text { Media CON }\end{array}$ & $\begin{array}{lr}\text { Potencia Pot } \\
\text { Media ECC Medi }\end{array}$ & $\begin{array}{ll}\text { encia } & \text { Potencia } \\
\text { a Total } & \text { Pico CON }\end{array}$ & $\begin{array}{l}\text { Potencia } \\
\text { Pico ECC }\end{array}$ & & \\
\hline
\end{tabular}

\section{Evaluación unilateral}

Los datos de potencia, trabajo y duración de la repetición del test unilateral, y el porcentaje de asimetría entre NL y L se recogen en la Tabla 3, Tabla 4 y Figura 1. Tras el análisis del test de potencia unilateral en NL se encontraron mejoras significativas (pd».05) en la potencia media (concéntrica, excéntrica y total) (TE grande) y en la potencia pico en la fase concéntrica (TE grande) así como una reducción significativa en la duración total de la repetición (TE grande).

Por otro lado, todos los valores analizados en el test de potencia relativo a L presentaron mejoras significativas (pd».05; TE grande) con porcentajes desde el $32.05 \%$ hasta el $125.52 \%$.

Respecto al test de asimetría, los resultados del post-test muestran una reducción en la asimetría entre ambas piernas en todas las variables de la potencia estudiadas (pico y media) y en todas las fases del movimiento, alcanzando en todas las variables analizadas valores de asimetría inferiores a los considerados de riesgo $(>15 \%)$

\section{Tiempo de baja}

La lesión del LLI de la rodilla produjo que el futbolista permaneciera de baja durante 36 días antes de incorporarse al trabajo con el grupo.

\section{Discusión}

Este estudio de caso se llevó a cabo para conocer el efecto de un programa de entrenamiento de fuerza con carga excéntrica ejecutado con dispositivos de inercia rotacional, sobre la potencia del tren inferior de un jugador con lesión LLI de la rodilla. También se pretendió analizar si este estímulo es efectivo en la reducción de las asimetrías causadas por la lesión. Los resultados obtenidos muestran que tras la aplicación del programa de entrenamiento el futbolista incrementó los niveles de potencia (bilateral y unilateral) y redujo las asimetrías obtenidas en pretest.

Los resultados obtenidos tras el proceso de intervención mostraron mejoras significativas en la potencia media. Esto coincide con los resultados de estudios previos en los que se aplicó una intervención basada en un programa de entrenamiento de fuerza con carga excéntrica a través de un dispositivo de inercia rotacional a un futbolista profesional durante el proceso de recuperación de una lesión de LCA(Núñez et al., 2016). Además, existen estudios previos (De Hoyo et al., 2015; Tous-Fajardo, Gonzalo-Skok, Arjol-Serrano \& Tesch, 2016) en los que tras la aplicación de un programa de entrenamiento de fuerza con carga excéntrica en dispositivo de inercia rotacional los futbolistas mejoraron diferentes variables determinantes del rendimiento (salto vertical y cambio de dirección) estrechamente relacionadas con el nivel de potencia del tren inferior (Swinton, Lloyd, Keogh, Agouris, \& Stewart, 2014). Las mejoras de la potencia media se produjeron en el test bilateral y en el unilateral para ambas piernas, aunque los mayores porcentajes de mejora tuvieron lugar en L. Esto puede ser debido a que esta extremidad presenta en pre-test valores de potencia media más bajos, que le hacen ser más receptivos al estímulo de entrenamiento. Bajos niveles en una capacidad han demostrado aumentar la sensibilidad ante los entrenamientos de mejora dirigidos a su desarrollo (Dawson et al., 1998).

Respecto a la potencia obtenida en las diferentes fases del movimiento, las ganancias fueron mayores en la fase concéntrica que en la excéntrica. Estos resultados son contrarios a los esperados, ya que la mayor actividad electromiográfica producida en la fase excéntrica como consecuencia de la carga generada por los medios de inercia rotacional empleados, debería haber incrementado más la potencia durante la fase de frenado que durante la propulsiva (Norrbrand, Tous-Fajardo, Vargas $\&$ Tesch, 2011). Es posible que el entrenamiento específico en campo realizado durante la última fase del proceso de readaptación explique el comportamiento de esta variable durante el post-test. Por otra parte, durante el entrenamiento no se utilizaron medios de monitorización de la carga en tiempo real, por lo que no es posible asegurar que se haya generado sobrecarga en la fase excéntrica durante las ejecuciones.

El incremento significativo del pico de potencia en $L$ durante la fase 
concéntrica y la fase excéntrica $(100.90 \%$ y $86.12 \%$, respectivamente), parece demostrar el efecto del entrenamiento con dispositivos de inercia rotacional sobre la capacidad de generar picos de potencia más elevados en la pierna afectada. Este resultado coincide con las mejoras relativas al pico de potencia encontradas en el trabajo de Núñez et al. (2016) con un futbolista lesionado del LCA.

El descenso en el tiempo de repetición en la ejecución del squat lateral (-46.24\%) podría estar relacionado con un incremento de la potencia en L en ambas fases del movimiento (Núñez et al., 2016), debido principalmente a la capacidad del ejercicio squat de movilizar las fibras musculares rápidas de los grupos musculares implicados en dicha acción. Además, este incremento de la potencia llevó asociado un aumento significativo de la energía necesaria para desplazar el volante de inercia, tanto en la ejecución bilateral (29.95\%) como en la ejecución unilateral de PL (32.05\%). Estos resultados difieren de los obtenidos en el trabajo de Núñez et al. (2016), en el que a pesar del aumento de la potencia en el squat bilateral el cambio en la capacidad de trabajo fue prácticamente nulo.

En relación con las asimetrías de potencia entre NL y L, nuestros resultados presentaron reducciones sustanciales de las mismas (Figura 1). Tras el pre-test todos los valores de potencia analizados presentaron valores de asimetría calificados como de alto riesgo $(>15 \%)$ (Petisco et al., 2016). Por el contrario, tras el proceso de intervención las diferencias entre ambas piernas se vieron reducidas a valores que oscilan entre el $.38 \%-7.02 \%$. Esta evolución provoca una disminución del riesgo de lesión debido al déficit de potencia bilateral. Esta reducción de la asimetría entre ambas piernas puede verse influenciada por la inclusión de ejecuciones de carácter unilateral (Gonzalo-Skok, Tous-Fajardo, SuárezArrones, Arjol-Serrano, Casajús, \& Méndez-Villanueva, 2017). Sin embargo, con nuestro futbolista lesionado sometido a un programa de entrenamiento que incluye ejercicios de orientación bilateral y unilateral, no podemos concluir que esta reducción se deba únicamente al entrenamiento unilateral.

Tras la aplicación del programa de entrenamiento de fuerza con carga excéntrica propuesto en este trabajo, el jugador se reincorporó al trabajo con el grupo 36 días después de sufrir la lesión, realizando durante esa semana de trabajo grupal las últimas tres sesiones de entrenamiento de fuerza con sobrecarga excéntrica. Según el estudio epidemiológico de Lundblad et al. (2013), el tiempo medio de recuperación de un esguince del LLI de la rodilla es de $23 \pm 23$ días de baja. En el caso de las lesiones de grado II de esta misma estructura los días de baja pueden superar este tiempo de recuperación y alcanzar valores de $46 \pm$ 10 días hasta la incorporación al entrenamiento normalizado (Lundblad et al., 2013). La reducción de los días de baja junto a la corrección de las asimetrías entre NL y L nos lleva a pensar que el entrenamiento llevado a cabo en el dispositivo de inercia rotacional en comparación con los dispositivos tradicionales, produce un estímulo óptimo que lo convierte en una estrategia de gran valor dentro de los programas de readaptación física-deportiva que pretenden optimizar las ganancias de potencia muscular en el futbolista lesionado.

Una posible limitación de este estudio ha sido la falta de monitorización del entrenamiento realizado, que habría permitido dar feedback a la ejecución del deportista, cuantificar el estímulo de entrenamiento y observar la presencia de sobrecarga excéntrica durante la ejecución.

\section{Conclusiones}

La inclusión de un programa de entrenamiento de fuerza con carga excéntrica ejecutado en un dispositivo de inercia rotacional como parte principal del proceso de readaptación físico-deportiva de una lesión del LLI de la rodilla de un futbolista se ha mostrado como un método eficaz para incrementar la potencia muscular del tren inferior tanto en L como en NL. Estas mejoras en la potencia se han visto reflejadas en la reducción de asimetrías entre ambas piernas, permitiendo la disminución de la duración del proceso de recuperación.

\section{Referencias}

Ade, J.; Fitzpatrick, J., \& Bradley, P.S. (2016). High-intensity efforts in elite soccer matches and associated movement patterns, technical skills and tactical actions. Information for position-specific training drills. Journal of Sports Sciences, 34(24):2205-2214. https://doi.org/ 10.1080/02640414.2016.1217343

Andersen, L.L.; Andersen, J.L.; Magnusson, S.P.; Suetta, C.; Madsen, J.L. \& Christensen, L.R. (2005). Changes in the human muscle force-velo city relationship in response to resistance training and subsequent detraining. Journal of Applied Physiology, 99(1):87-94.

Askling, C.; Karlsson, J., \& Thorstensson,A. (2003). Hamstring injury occurrence in elite soccer players after preseason strength training with eccentric overload. Scandinavian Journal of Medicine in Science and Sports, 13(4):244-250.

Carling, C.; Bloomfield, J., Nelsen, L., \& Reilly, T. (2008). The role of motion analysis in elite soccer: contemporary performance measurement techniques and work rate data. Sports Medicine, 38(10):839-62.

Cohen, J.(1988). Statistical power analysis for the behavioural sciences. Hillside, NJ: L. Erbraum Associates.

Dawson, B.; Fitzsimons, M.; Green, S.; Goodman, C.; Carey, M., \& Cole, K. (1998). Changes in performance, muscle metabolites, enzymes and fibre types after short sprint training. European Journal of Applied Physiology, 78:163-169.

De Hoyo, M.; Pozzo, M.; Sañudo, B.; Carrasco, L.; Gonzalo-Skok, O.; Domínguez-Cobo, S., \& Morán-Camacho, E. (2015). Effects of a 10 -week in-season eccentric-overload training program on muscleinjury prevention and performance in junior elite soccer players. International Journal of Sports Physiology and Performance, 10(1):46-52. https://doi.org/10.1123/ijspp.2013-0547

Ekstrand, J.; Hägglund, M., \& Waldén, M. (2011). Epidemiology of muscle injuries in professional football (soccer). The American Journal of Sports Medicine, 39(6):1226-1232. https://doi.org/ $10.1177 / 0363546510395879$

Fousekis, K.; Tsepis, E., \& Vagenas, G. (2010). Lower limb strength in professional soccer players: profile, asymmetry, and training age. Journal of Sport Science and Medicine, 9(3):364-373.

Gabbett, T.J. (2016). The training-injury prevention paradox: should athletes be training smarter and harder? British Journal of Sports Medicine, 50(5):273-280. https://doi.org/10.1136/bjsports-2015095788

Gonzalo-Skok, O.; Tous-Fajardo, J.; Suarez-Arrones, L.; Arjol-Serrano, J.L.; Casajús, J.A., \& Mendez-Villanueva, A. (2017). SingleLeg Power Output and Between-Limbs Imbalances in Team-Sport Players: Unilateral Versus Bilateral Combined Resistance Training. International journal of sports physiology and performance, 12(1): 106-114. https://doi.org/ 10.1123/ijspp.2015-0743

Gustavsson, A.; Neeter, C.; Thomeé, P.; Silbernagel, K.G.; Augustsson, J.; Thomeé, R., \& Karlsson, J. (2006). A test battery for evaluating hop performance in patients with an ACL injury and patients who have undergone ACL reconstruction. Knee Surgery Sports Traumatology Arthroscopy, 14(8): 778-788.

Hägglund, M.; Waldén, M.; Bahr, R., \& Ekstrand, J. (2005). Methods for epidemiological study of injuries to professional football players: developing the UEFA model. British journal of sports medicine, 39(6): 340-346.

Hoffman, J. R.; Ratamess, N. A.; Klatt, M.; Faigenbaum, A. D., \& Kang, J. (2007). Do bilateral power deficits influence directionspecific movement patterns? Research in Sports Medicine, 15: 1-8.

Lalín, C. (2008). La readaptación lesional (II parte): reentrenamiento físico deportivo del deportista lesionado. RED: Revista de entrenamiento deportivo, 3: 29-37.

Lundblad, M.; Waldén, M.; Magnusson, H.; Karlsson, J., \& Ekstrand, J. (2013). The UEFA injury study: 11-year data concerning 346 MCL injuries and time to return to play. British Journal of Sports 
Medicine, 47(12):759-762. https://doi.org/10.1136/bjsports-2013092305

Macmahon, P. Current diagnosis \& treatment in sports medicine. New York: McGraw Hill Medical Pub; 2007.

Menzel, H.J.; Chagas, M.H.; Szmuchrowski, L.A.; Araujo, S.R.; De Andrade, A.G., \& De Jesus-Moraleida, F.R. (2013). Analysis of lower limb asymmetries by isokinetic and vertical jump tests in soccer players. Journal of Strength \& Conditioning Research, 27(5):1370-1377.https://doi.org/10.1519/JSC.0b013e318265a3c8

Miyamoto, R.G.; Bosco, J., \& Sherman, O. (2009). Treatment of Medial Collateral Ligament Injuries. The Journal of the American Academy of Orthopaedic Surgeons, 17(3):152-61.

Norrbrand, L.; Tous-Fajardo, J.; Vargas, R., \& Tesch, P.A. (2011). Quadriceps muscle use in the flywheel and barbell squat. Aviation Space Environmental Medicine, 82:13-19.

Noya, J.; Gómez-Carmona, P.M.; Gracia-Marco, L.; Moliner-Urdiales, D., \& Sillero-Quintana, M. (2014). Epidemiology of injuries in First Division Spanish football. Journal of Sports Sciences, 32(13): 1263-1270. https://doi.org/10.1080/02640414.2014.884720

Núñez, V.; Lancho, C., \& Ramírez, J.M. (2016). Entrenamiento muscular a través de tecnología isoinercial en un jugador de fútbol profesional intervenido de rotura total de LCA. Estudio de caso. RETOS. Nuevas Tendencias en Educación Física, Deporte y Recreación, 29:166-170.

Owen, A.; Wong, D.P.; McKenna, M., \& Dellal, A. (2011). Heart rate responses and technical comparison between small-vs. large-sided games in elite professional soccer. Journal of Strength \& Conditioning Research, 25(8):2104-2110. https://doi.org/10.1519/ JSC.0b013e3181f0a8a3

Owen, A.; Wong, P.; Dellal, A.; Paul, D.J.; Orhant, E., \& Collie, S. (2013). Effect of an injury prevention program on muscle injuries in elite professional soccer. Journal of Strength \& Conditioning Research, 27(12):3275-3285. https://doi.org/10.1519/ JSC.0b013e318290cb3a

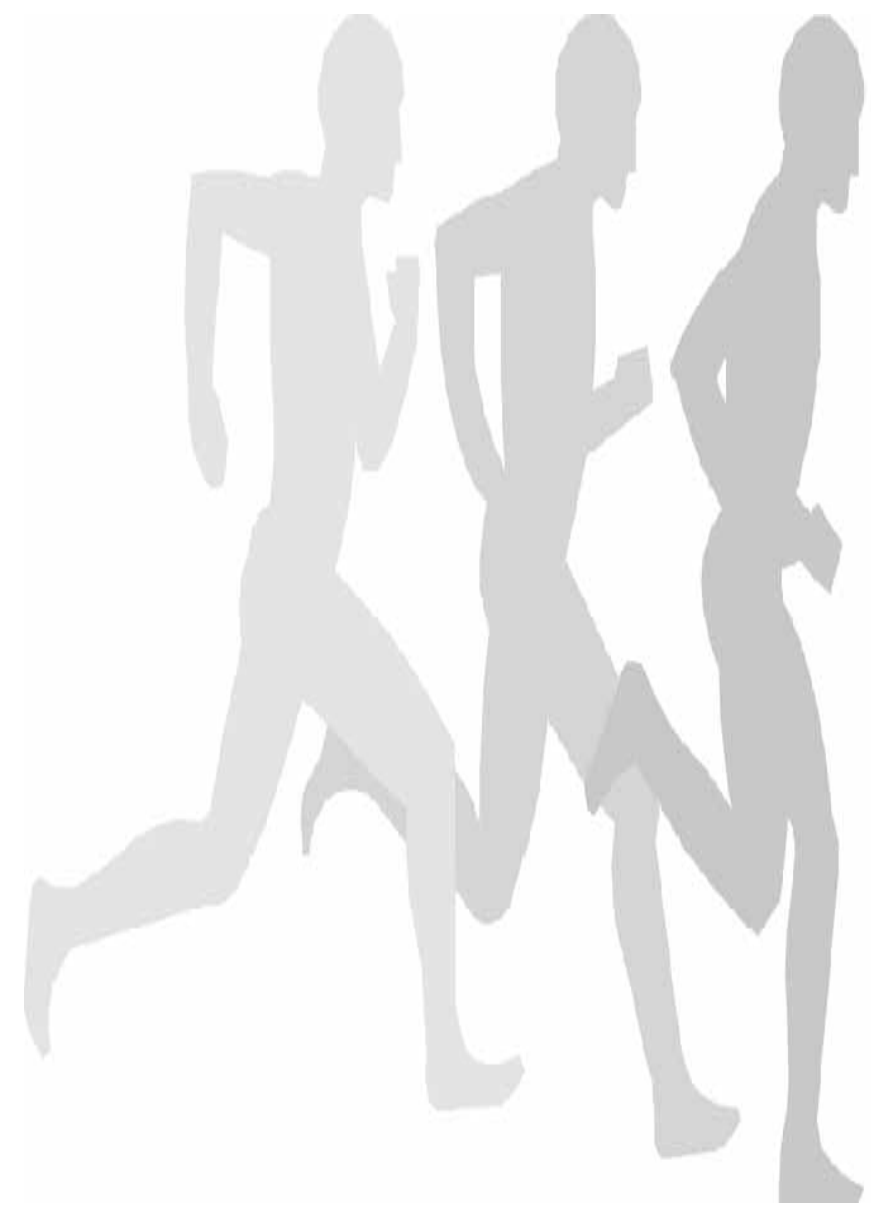

Petisco, C.; Carretero, M., \& Sánchez-Sánchez, J. (2016). Is exercise a determining factor of functional asymmetries in the lower limb? Apunts. Educación Física y Deportes, 125:7-20.

Phisitkul, P.; James, S.L.; Wolf, B.R., \& Amendola, A. (2006). MCL injuries of the knee: current concepts review. The Iowa Orthopaedic Journal, 26:77-90.

Romero, S.; Feria,A.; Sañudo, B.; De Hoyo, M., \& Del Ojo, J.J. (2014). Efectos de entrenamiento de fuerza en sistema isoinercial sobre la mejora del CMJ en jóvenes futbolistas de elite. RETOS. Nuevas Tendencias en Educación Física, Deporte y Recreación, 26:180182.

Romero-Rodriguez, D.; Gual, G., \& Tesch, P.A. (2011). Efficacy of an inertial resistance training paradigm in the treatment of patellar tendinopathy in athletes: a case-series study. Physical Therapy in Sport, 12(1):43-48. https://doi.org/10.1016/j.ptsp.2010.10.003

Ronconi, M., \& Alvero-Cruz, J.R. (2008). Cambios fisiológicos debidos al desentrenamiento. Apunts Medicina de L'Esport, 160:1928.

Swinton, P.; Lloyd, R.; Keogh, R.; Agouris, I., \& Stewart,A.D. (2014). Regression models of sprint, vertical jump, and change of direction performance. Journal of Strength and Conditioning Research, 28(7):1839-1848. https://doi.org/10.1519/ JSC.0000000000000348

Tous-Fajardo, J.; Maldonado, R., Quintana, J.M., Pozzo, M., \& Tesch, P.A. (2006). The flywheel leg-curl machine: offering eccentric overload for hamstring development. International Journal of Sports Physiology and Performance 1(3):293-299.

Tous-Fajardo, J.; Gonzalo-Skok, O.; Arjol-Serrano, J.L., \& Tesch, P. (2016). Enhancing Change-of-Direction Speed in Soccer Players by Functional Inertial Eccentric Overload and Vibration Training. International Journal of Sports Physiology and Performance, 11(1):66-73. https://doi.org/10.1123/ijspp.2015-0010

Van Winckel J, Helsen W, McMillan K, Tenney D, Meert JP, Bradley P. Fitness in soccer: the science and practical application. KleinGelmen: Moveo Ergo Sum; 2014.

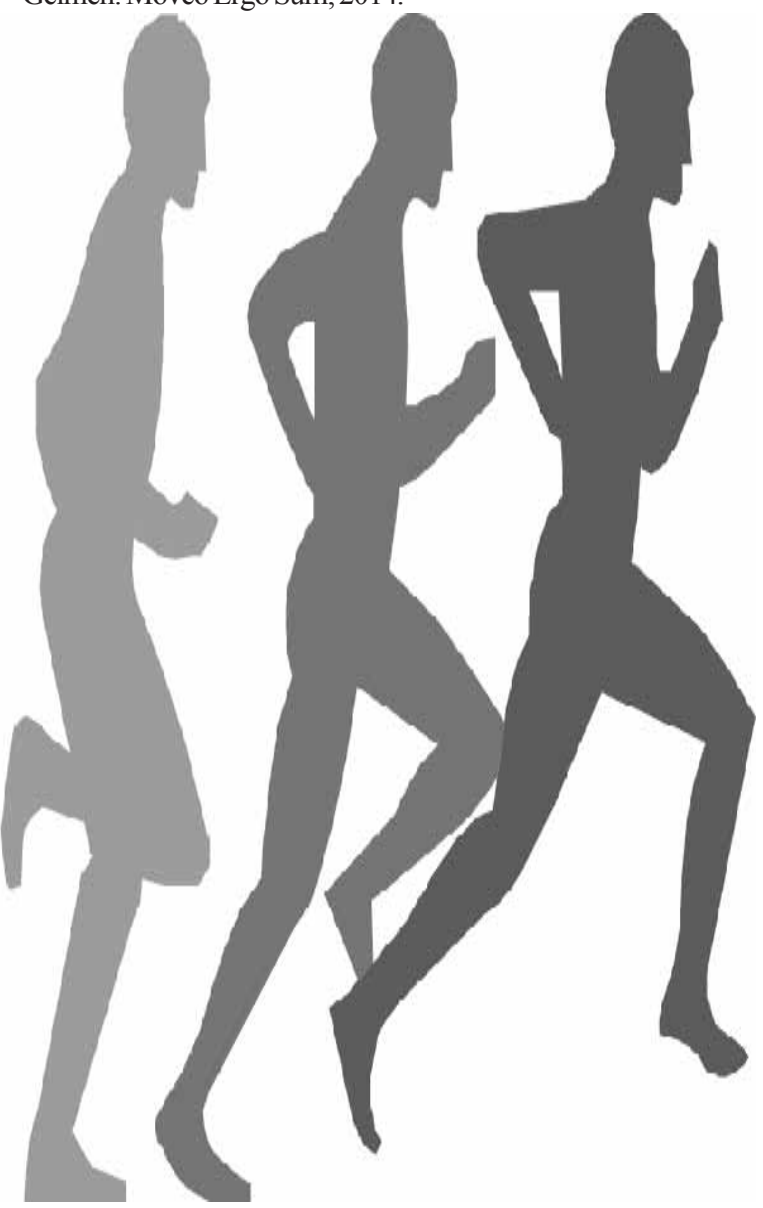

